\title{
MUTIS Y LA INTRODUCCIÓN DEL MÉTODO ANALÍTICO EN COLOMBIA ${ }^{\alpha}$
}

\author{
Mutis and the Introduction of Analytic Method in Colombia
}

\author{
Luis Carlos Arboleda ${ }^{\beta}$
}

Fecha de recepción: 28/02/2019 • Fecha de aceptación: 28/05/2019

Resumen. En este trabajo se estudian las distintas modalidades de enseñanza del método analítico por José Celestino Mutis (1732-1808) en la cátedra de matemáticas del Colegio del Rosario de Bogotá durante la segunda mitad del siglo XVIII. Como marco de referencia para apreciar mejor las transformaciones de esta enseñanza, se empezará por recordar algunas de las características históricas y filosóficas más importantes de la distinción cartesiana entre análisis y síntesis en el pensamiento matemático. Mutis empieza introduciendo el enfoque logicista del Discurso del Método de Wolff. Luego explica las reglas cartesianas del análisis y la síntesis, para lo cual traduce fragmentos de los Comentarios a la geometría de Descartes de Rabuel. También presenta las ideas de Newton sobre el método analítico, tanto en la modalidad experimental de la Óptica como en la perspectiva físico-matemática de los Principia. Se analizará el esfuerzo que pudo haber significado la comprensión de este cambio de perspectiva en el proceso de lectura y traducción de los Principia por parte de Mutis. Enseguida se muestra que al centrarse la enseñanza en los Elementos de Matemáticas de Bails - como consecuencia de la reforma del plan de estudios de la cátedra promovida por Mutis-, se termina adoptando la modalidad operatoria del análisis como aplicación del álgebra a la geometría. Finalmente se muestra que la introducción del texto de Bails conllevó una transformación de fondo en el enfoque epistemológico, cognitivo y pedagógico de la enseñanza por el método analítico en la cátedra del Rosario, comparada con los Elementa Matheseos Universae de Wolff en su etapa fundacional.

\footnotetext{
${ }^{\alpha}$ Una versión de este trabajo se presentó en la conferencia paralela «Introducción histórica del método analítico en la enseñanza de las matemáticas en Colombia». XV Conferencia Interamericana de Educación Matemática-CIAEM, Medellín, Colombia, 5-10 de mayo de 2019.

B Grupo de Historia y Filosofía de la Práctica Matemática, Instituto de Educación y Pedagogía, Universidad del Valle, Cali, Colombia. luis.carlos.arboleda@gmail.com (DD https://orcid.org/0000-0003-0444-1383
} 
Palabras clave: Historia de la educación matemática; Enseñanza del método analítico; José Celestino Mutis; Historia del Colegio del Rosario de Bogotá.

Abstract. In this work we study the different ways of teaching the analytical method by José Celestino Mutis (1732-1808) as the chair of mathematics at the Colegio del Rosario in Bogotá during the second half of the 18th century. As a framework to better appreciate the transformations of this teaching, we will begin by recalling some of the most important historical and philosophical characteristics of the Cartesian distinction between analysis and synthesis in mathematical thought. Mutis begins by introducing the logicist approach of Wolff's Discourse on Method. He then explains the Cartesian rules of analysis and synthesis, for which he translates fragments of Rabuel's Commentaries on Descartes' geometry. He also presents Newton's ideas on the analytical method, both in the experimental modality of Optics and in the physical-mathematical perspective of Principia. We will analyze the difficulty inherent to the understanding of this change of perspective in the process of the reading and translation of Principia by Mutis. Then it is shown that by focusing the teaching on Bails' Elementos de Matemáticas - as a consequence of the reform of the curriculum of the chair promoted by Mutis-, it ends up adopting the operative mode of analysis as an application of algebra to geometry. Finally, we show how the introduction of the Bails text entailed a fundamental transformation in the epistemological, cognitive and pedagogical approach to teaching by the analytical method in the Cátedra del Rosario, as compared to Wolff's Elementa Matheseos Universae in its foundational stage.

Keywords: History of mathematics education; Teaching of analytical method; José Celestino Mutis; History of the Colegio del Rosario in Bogota

\section{INTRODUCCIÓN}

En este trabajo se analiza la recepción, circulación, uso y apropiación de nociones relativas al método analítico en uno de los momentos históricos decisivos de la enseñanza moderna de las matemáticas en Colombia y, al mismo tiempo, en uno de los establecimientos emblemáticos en la institucionalización de esta enseñanza: la cátedra de Matemáticas del Colegio del Rosario.

Este momento corresponde a la divulgación de la ciencia colonial ilustrada entre las élites criollas del virreinato de la Nueva Granada en la segunda mitad del siglo XVIII. El catedrático José Celestino Mutis 
promueve inicialmente un enfoque logicista de la formación matemática basado en el Discurso del método de Wolff. Luego explica las reglas cartesianas de análisis y síntesis como parte de sus lecciones de geometría analítica. Llama entonces la atención sobre la necesidad de distinguir el método matemático y la geometría algebraica de Descartes, de su orientación escolástica y su filosofía de sistema.

En un ambiente ideologizado, dominado por las polémicas con las comunidades religiosas que se resistían a la divulgación local de las ideas de Copérnico y Newton, Mutis enseña los fundamentos de la nueva física. En estas circunstancias el discurso sobre el método matemático abandona la perspectiva logicista de Wolff y Mutis con sus alumnos más destacados se consagran a interpretar el uso que le da Newton a los procedimientos de análisis y síntesis en el estudio de los fenómenos de la mecánica racional.

Un evento excepcional producto de esta etapa es la traducción inédita e incompleta de los Principia de Newton, la primera en castellano por muchos años. Las reformas de los planes de estudio con lo traumáticas e inestables que fueron, favorecieron en todo caso transformaciones en el pensamiento local del método analítico a través del uso y apropiación en la enseñanza de tratados de matemáticas como los Elementos de Matemática de Bails, con un enfoque epistemológico, cognitivo y pedagógico diferente a un curso tradicional como el de Wolff.

Para mejor apreciar los alcances de la enseñanza del método analítico por Mutis en la cátedra de matemáticas del Rosario, es conveniente hacer antes algunas consideraciones generales sobre la importancia de esta cuestión en las transformaciones de la práctica matemática en la segunda mitad del siglo XVIII y comienzos del XIX. Por la misma razón se hace necesario fijar las concepciones de Pappus y Descartes sobre la distinción entre lo analítico y lo sintético en el pensamiento matemático.

\section{EL MÉTODO ANALÍTICO SEGÚN PAPPUS Y DESCARTES}

La distinción entre análisis y síntesis tiene una larga y compleja historia en las matemáticas y la filosofía. Michael Otte y Marco Panza han formulado una propuesta de clasificación de las modalidades bajo las cuales ha sido abordada esta relación: fenomenológica, genética, 
representacional, pragmática, programática, direccional, configuracional, lógico-teórica, lingüística y disciplinaria. ${ }^{1}$ El propósito central de discutir estas interpretaciones radica en el problema histórico-filosófico que se encuentra en el fondo de la cuestión, esto es, el problema de la objetividad matemática como forma de conocimiento. Así pues, la importancia de estudiar la distinción análisis-síntesis para estos autores radica en mejorar nuestra comprensión sobre la complementariedad entre medios y objetos de la matemática como actividad de razonamiento humano. $^{2}$

Se sostiene que el creciente alejamiento de las ciencias naturales del enfoque tradicional de las matemáticas que se puso en vigor en la segunda mitad del siglo XVIII, no podría entenderse si no se tiene en cuenta el desarrollo complejo de la experiencia y la práctica científica como consecuencia del incremento en las innovaciones en los métodos, técnicas y teorías, así como los cambios en el procesamiento de la información y la organización espacial del conocimiento. Por otro lado, en los mismos años se dieron transformaciones sustanciales en las formas de pensamiento matemático. Hacia comienzos del siglo XIX el pensamiento relacional ya había operado rupturas radicales con el pensamiento tradicional de las sustancias en tanto sujeto de predicación. La ciencia de las formas se alejó de la ciencia de los fenómenos, y las teorías empezaron a ser consideradas como realidades sui generis; evidentemente teniendo en cuenta que toda teoría corresponde a un modelo del mundo real y, en consecuencia, es el producto de una actividad subjetiva.

En los orígenes más directos de este pensamiento relacional se encontraban las innovaciones del «cálculo geométrico» introducido por Descartes en la Geometría ${ }^{3}$ con el propósito de establecer la distinción entre geometría y álgebra. Tales innovaciones — representadas en técnicas algebraicas para analizar problemas geométricos, conectar la construcción de curvas con sus respectivas ecuaciones algebraicas y clasificar las curvas según el grado de las ecuaciones que las representan-, resultan de la

\footnotetext{
1 Ver la introducción de los compiladores en Michael Otte y Marco Panza (eds.), Analysis and Synthesis in Mathematics (Dordrecht: Kluwer, 1997), ix-xiii.

2 Otte y Panza, Analysis and Synthesis, xii.

${ }_{3}^{3}$ Ver la edición facsimilar de la Géométrie de 1637 con traducción al inglés en René Descartes, The Geometry of René Descartes with a facsimile of the first edition (New York: Dover, 1954).
} 
fecunda aplicación del método de análisis y síntesis a la solución del siguiente problema de Pappus:

Encontrar el lugar de los puntos tales que, si a partir de cada uno de ellos se trazan rectas que se cortan en ángulos dados, respectivamente con otras cuatro rectas dadas en posición, el producto de los dos segmentos que van desde el punto a dos de estas rectas, es igual al producto de los dos segmentos que van desde el punto a las otras dos. ${ }^{4}$

Recordemos que la distinción analítico-sintético se origina en la filosofía griega. Esta tradición se revela en el siguiente escolio al libro 13 de los Elementos de Euclides: «El análisis, entonces, consiste en tomar como admitido lo que se busca para llegar por vía de consecuencia a algo cuya verdad ya ha sido admitida, mientras que la síntesis consiste en tomar algo admitido y pasar por vía de consecuencia a algo admitido como verdad».5

Por otra parte, es al comienzo del libro 7 de sus Mathematicae Collectiones (traducción de Comandino de 1589) que Pappus formula sus ideas sobre las nociones de análisis-síntesis, ${ }^{6}$ las cuales usualmente se interpretan en los siguientes términos:

En el análisis, al suponerse que lo que se busca ya ha sido obtenido, examinamos aquello de donde procede y de nuevo las premisas de donde esto procede, hasta que remontamos de esta manera a algo ya conocido o que cumple la función de principio. A la inversa, en la síntesis se supone ya obtenido lo que en el análisis se busca como último término. Colocando en el orden natural (deductivo) los antecedentes del análisis en lugar de consecuentes, y relacionando unos y otros, llegamos a la meta que es la construcción del objeto buscado. ${ }^{7}$

\footnotetext{
${ }^{4}$ Ver la formulación y el estudio del problema de Pappus, por ejemplo, en Luis Carlos Arboleda, «El análisis cartesiano en la solución del problema de Pappus y la introducción de las curvas algebraicas», en Asociación Colombiana de Matemática Educativa $13^{\circ}$ Encuentro, ed. Gilberto Obando Zapata (Medellín: Editorial Universidad de Medellín, 2013), 764-776. La formulación original del problema de las cuatro líneas de Pappus se encuentra en Descartes, Geometry, 27.

5 Jacob Klein, Greek Mathematical Thought and the Origin of Algebra (New York: Dover, 1968), 259.

${ }^{6}$ Klein, Greek Mathematical Thought and the Origin of Algebra, 260.

7 Jaakko Hintikka y Unto Remes, The Method of Analysis. Its Geometrical Origin and Its General Significance. (Dordrecht: Reidel, 1974), 8-10.
} 
El esquema lógico del método de análisis y síntesis propuesto por Hintikka y Remes ${ }^{8}$ e interpretado por Gardies, ${ }^{9}$ es el siguiente:

1. Enunciar aquello que nos es dado: $D\left(D^{\prime}, D^{\prime \prime}\right.$ partes de $\left.D\right)$.

2. Enunciar aquello que se busca: $z$

1. La etapa del Análisis: $D^{\prime} \Rightarrow(z \Rightarrow d)$.

2. La etapa de la resolución: $(d \Rightarrow D)$

3. La etapa de la construcción: Fin del Análisis; inicio de la Síntesis.

4. Demostración por medio de la síntesis: $D^{\prime \prime} \Rightarrow(d \Rightarrow z)$.

Los resultados 6 y 3 permiten concluir que se llega a una equivalencia lógica entre $z$ y $d$, cuando se parte de lo que nos es dado $D$ o de sus partes $D^{\prime}$ y $D^{\prime \prime}$. Observemos también que la etapa 3 del análisis se inicia cuando a partir de una parte de lo dado y considerando lo buscado como dado, se deduce una afirmación que, en virtud de la resolución, se comporta como principio de lo dado.

La caracterización cartesiana de la relación análisis-síntesis en la Geometría es la siguiente:

$\mathrm{Si}$, entonces, deseamos resolver cualquier problema, suponemos primero que la solución ya se ha efectuado y damos nombres a todas las líneas que parecen necesarias para su construcción, tanto a las desconocidas como a las que se conocen. Luego, sin hacer distinciones entre líneas conocidas y desconocidas, debemos desentrañar la dificultad de una manera que muestre lo más naturalmente posible las relaciones entre estas líneas, hasta que encontremos posible expresar una única cantidad de dos maneras. Esto constituirá una ecuación, ya que los términos de una de estas dos expresiones son uno y otro iguales a los términos de la otra. ${ }^{10}$

Anotemos finalmente que en su reconstrucción del procedimiento analítico empleado para la solución del problema de Pappus en los

\footnotetext{
8 Hintikka y Remes, The Method of Analysis, 22-26.

9 Jean-Louis Gardies, Qu'est-ce que et pourquoi l'analyse? Essai de définition (Paris: Vrin, 2001), 28-29.

${ }_{10}$ Descartes, Geometry, 6-9.
} 
libros 1 y 2 de la Geometría, Gardies considera que Descartes utiliza dos modalidades de análisis. La primera consiste en aceptar como dado el lugar geométrico de los puntos que aportan solución al problema, para remontarse a partir de allí a la ecuación general de segundo grado como principio. La segunda modalidad de análisis consiste en remontarse del principio (la ecuación general) a otros principios, las ecuaciones restringidas de cada uno de los lugares geométricos que representan la solución. No obstante, Descartes no aporta la deducción lógica de cada lugar geométrico a partir de su ecuación, limitándose a exhibirlo como dado mediante una construcción. ${ }^{11}$

\section{EL DISCURSO WOLFFIANO DEL MÉTODO MATEMÁTICO Y LA INSTAURACIÓN DE LA CÁTEDRA DE MUTIS}

La creación de la cátedra de Matemáticas en el Colegio del Rosario de Santafé de Bogotá (1762) marca un momento decisivo en la introducción en Colombia de una enseñanza de las matemáticas dentro de los cánones del pensamiento ilustrado. El fundador y encargado de la cátedra por varios decenios fue José Celestino Mutis (1732-1808), un joven médico del virrey Mecía de la Cerda, que llegó al país dotado de una sólida formación en la nueva ciencia (botánica, física, astronomía y matemáticas) obtenida en Cádiz y Madrid, y que con el tiempo se convertiría en el director de la célebre Expedición Botánica de la Nueva Granada (1783) y del Observatorio astronómico (1803), el primero en América.

El 13 de marzo de 1762 Mutis pronuncia el Discurso preliminar o de inauguración de la cátedra en el cual expone sus ideas sobre la utilidad práctica de las matemáticas y su importancia en todo pensamiento racional sobre la naturaleza. ${ }^{12}$ Nos interesa destacar el tratamiento que da Mutis en este discurso a lo que él denomina los «métodos sintético y analítico», según la expresión de Christian Wolff

\footnotetext{
${ }^{11}$ Gardies, Qu'est-ce que et pourquoi l'analyse?, 107-116.

12 Ver el texto del Discurso, por ejemplo, en: José Celestino Mutis, Pensamiento científico y filosófico de José Celestino Mutis, ed. Gonzalo Hernández de Alba (Bogotá: Fondo Cultural Cafetero, 1982), 33-42. Nuestro estudio del documento se encuentra en Luis Carlos Arboleda, «Matemáticas, Cultura y Sociedad en Colombia», en Historia Social de la Ciencia en Colombia, ed. Emilio Quevedo (Bogotá: Colciencias, 1993), t. II, 13-172.
} 
el autor de los Elementa Matheseos Universae, obra de referencia en los primeros años de la cátedra. ${ }^{13}$

En primer lugar, se trata de explicar la conexión de estos métodos con el modo lógico en que se expresa el entendimiento de las cosas: «[En las matemáticas] se acostumbra el entendimiento a proceder sin error, conduciéndose siempre de unas verdades a otras, de la más simple hasta la más compuesta, o al contrario, según la aplicación de los dos métodos sintético y analítico». ${ }^{14}$

Con el fin de aclarar la secuencia de los términos sintético-analítico, Mutis anota a continuación que la geometría (euclidiana) es la parte de las matemáticas en donde se observa este «ajustado método de proceder» (sintético).

Luego, en su lección sobre el «Método matemático», se dedica a precisar algunos aspectos del método expuestos de manera general en la inauguración: la naturaleza y características del discurso matemático, su constitución, organización, lenguaje y criterios de validación interna. En un trabajo anterior ${ }^{15}$ hemos mostrado que Mutis se basa esencialmente en el enfoque logicista del Discurso del método que antecede los Elementa de Wolff.16

Al inicio de la lección Mutis afirma: «Todo el artificio de las matemáticas, su certidumbre y solidez consisten en el admirable orden de que usan los matemáticos para enseñar sus dogmas». Este orden del método geométrico se desenvuelve en dos componentes, resolución (análisis) y demostración (síntesis):

\footnotetext{
${ }_{13}$ Christian Wolff, Elementa matheseos universae (Halle: Magdeburgicae, 1713-1715). La edición consultada por Mutis, la cual se conserva en la Biblioteca Nacional de Colombia, es la siguiente: Christian Wolff, Elementa matheseos universae. Editio novissima, multo auctior et Correctior, 5 tomos. (Genevae: Henricum-Albertum Gosse, 1743-1752). Ver la traducción francesa aumentada: Christian Wolff, Cours de mathématique contenant toutes les parties de cette science mises à la portée des commençants (Paris: Jombert, 1757).

${ }_{14}$ Mutis, Pensamiento científico y filosófico, 36.

15 Arboleda, «Matemáticas, Cultura y Sociedad en Colombia», 45-62.

${ }^{16}$ Christian Wolff, «Discours Préliminaire sur la Méthode don't on se sert pour traiter les Mathématiques», en Wolff, Cours de mathématique, tomo I, i-xii.
} 
El orden con que se procede en las resoluciones y demostraciones es tan exacto y riguroso, que nada se admite, nada se deja pasar sin prueba. Ha merecido esta ciencia por la solidez que le es muy particular, calificar todo el método exacto en cualquier materia que sea. Y este modo de proceder los matemáticos es lo que se llama método geométrico. ${ }^{17}$

El enfoque wolffiano del método que aprendieron los primeros alumnos en la etapa fundacional de la cátedra se centró en los siguientes principios: el método matemático es universal y provee un conocimiento sólido de las cosas; forma hábitos de orden y exactitud de juicio que producen «una facilidad y una viveza admirable para percibir la verdad en otras ciencias a las que se aplica»; otras prácticas y ciencias «muy útiles al comercio de la vida» no podrían por ellas mismas, sin la intervención del método matemático, proporcionar «esa fuerza de imaginación, esa vivacidad y ese hábito de invención» que se obtienen a través del pensamiento deductivo; "hay dos métodos generales para buscar las verdades en las matemáticas, a saber la Síntesis y el Análisis».18

De acuerdo con Wolff, Mutis explica en su lección que el método matemático es el orden exacto y riguroso que los matemáticos utilizan en la presentación de sus conceptos. Primero las definiciones, seguidas de los axiomas y postulados en el caso de las matemáticas puras, o de experimentos y observaciones en las matemáticas mixtas y, finalmente, los teoremas y problemas. Establecer la verdad de una proposición consiste en demostrarla como teorema mediante una cadena de inferencias a partir de definiciones y axiomas. Este procedimiento se adelanta «observando cuidadosamente las reglas que proponen los lógicos para hacer sus silogismos» $\cdot{ }^{19}$ En este discurso wolffiano del orden la prioridad no reside en el axioma o postulado euclidiano sino en la definición, la cual, más allá de aclarar los conceptos, cumple un papel inferencial. Para Mutis un «teorema» es «una proposición teórica deducida de muchas definiciones comparadas entre sí».20

\footnotetext{
17 Mutis, Pensamiento científico y filosófico, 125.

18 Arboleda, «Matemáticas, Cultura y Sociedad en Colombia», 48-49.

19 Mutis, Pensamiento científico y filosófico, 132.

20 Mutis, Pensamiento científico y filosófico, 130.
} 
Como se observa, en el discurso wolffiano tal como Mutis lo interpreta y divulga en la Nueva Granada, la noción de orden tiene una connotación especial, en tanto dispositivo fundamentador de las matemáticas que, en el desarrollo del análisis de los conceptos, se expresa en categorías de principios bien determinadas. Es tal vez en esta dirección que apunta la afirmación de Paola Cantù ${ }^{21}$ de que el orden en Wolff correspondería a un cierto propósito de fundamentar el conocimiento en una jerarquía de conceptos básicos de aritmética, geometría y todas las demás ramas del conocimiento humano. Esta autora sugiere una conexión entre este punto de vista de Wolff, y la concepción de Hilbert de fundamentar la geometría en grupos de axiomas relativos a las nociones de incidencia, orden, congruencia, paralelismo y continuidad. Así mismo, la creencia de Wolff en la posibilidad de un análisis de conceptos para determinar las nociones primitivas entre todas las demás no estaría alejada de otras concepciones fundacionales del programa de Hilbert; por ejemplo, en cuanto a que la noción geométrica de semejanza sea jerárquicamente superior a la noción de congruencia y que, en consecuencia, todos los teoremas que dependen de la primera anteceden a aquellos que dependen de la segunda. ${ }^{22}$

Recordemos a este respecto el famoso epígrafe de Kant a los Fundamentos de la Geometría de Hilbert: «Todo conocimiento humano se inicia con intuiciones, pasa de éstas a los conceptos y termina en las ideas». ${ }^{23}$ Este epígrafe le permite a Hilbert resaltar el papel protagónico que juegan en su geometría aquellos "principios fundamentales» llamados axiomas. Pero, enseguida aclara que el sistema de axiomas de la geometría solo se establece como tal, a través del «análisis lógico de nuestra intuición espacial». Es el análisis lógico el que permite pasar de la intuición espacial —en donde los objetos aparecen como dados- a los principios que le sirven de fundamento a los conceptos e ideas geométricas. ${ }^{24}$

\footnotetext{
21 Paola Cantù, «Mathematics. Systematical Concepts», en Handbuch Christian Wolff, ed. Robert Theis y Alexander Aichele (Wiesbaden: Springer, 2018), 357-380.

22 Cantù, «Mathematics. Systematical Concepts», 373.

${ }^{23}$ Ver epígrafe e Introducción en: David Hilbert, The Foundations of Geometry (La Salle: The Open Court Publishing Co., 1950), 1.

${ }^{24}$ Ver sección «Grupo de axiomas» en Hilbert, The Foundations of Geometry, 2-17.
} 
Al comienzo de su lección del Colegio del Rosario sobre el método, Mutis explica la noción de análisis conceptual de los principios en términos de las tres reglas generales que fundamentan el método geométrico:

La primera es que de las ideas más sencillas y más generales se ha de subir a las más compuestas y menos generales. La segunda es que en la definición de los términos nada quede oscuro, nada quede ambiguo. La tercera es que todas las proposiciones, cuyas verdades no constan a primera vista por la significación y percepción de los mismos términos con que se enuncian, se hayan de probar demostrando muchas verdades y, por medio de las definiciones supuestas, los axiomas concedidos y las proposiciones ya demostradas..$^{25}$

Mutis pudo confirmar el rol de las definiciones en función del ideal filosófico de Wolff al enseñar sus lecciones de aritmética con base en el primer capítulo de los Elementa Matheseos. El manuscrito con la traducción de estas lecciones bajo el título de «Elementos de aritmética», fue identificado recientemente en el Fondo Mutis del Real Jardín Botánico de Madrid por Sebastián Molina, y es analizado a profundidad en su tesis doctoral junto con otros documentos de la cátedra. ${ }^{26}$ El carácter inferencial y al mismo tiempo filosófico de las definiciones ha debido hacérsele evidente, por ejemplo, en la traducción de la definición 2 del concepto de número uno:

Uno es aquello, que siendo algo no puede al mismo tiempo ser otra cosa dejando de ser lo mismo que es. Leibniz se explica en estos términos: supóngase por un instante que A es B, supóngase también que B no es D; supuesto esto si no se supone que A es D, vendremos en conocimiento de que $\mathrm{A}$ no es $\mathrm{B}$, sino uno diverso del otro llamado B. Y aplicando la definición nuestra a términos más precisos, diremos, que A es uno, porque siendo A que es ser algo, no puede ser B que sería ser otra cosa, diverso de A o de aquello mismo que es. ${ }^{27}$

\footnotetext{
25 Mutis, Pensamiento científico y filosófico, 125-126.

26 Sebastián Molina, "No hay reino que no sea newtoniano": José Celestino Mutis and the appropriation of Newton's experimental physics in New Granada (1762-1804)» (PhD diss., Università degli Studi di Torino, Turín, 2018), 103-118. Agradecemos al autor la gentileza de enviarnos una copia de su tesis.

${ }_{27}$ Molina, «José Celestino Mutis and the appropriation of Newton's experimental physics in New Granada», 109.
} 
Desde nuestra perspectiva de la aritmética formal esta traducción mutisiana aparece embarazosa y poco clara. Sin embargo, también sabemos que la dificultad epistemológica de esta definición para tratar de expresar la propiedad característica del concepto de «uno», radicaba en disponer para ello únicamente de los recursos limitados de la lógica de predicados, la única disponible en el contexto de la época. Por otra parte, el hecho de que Mutis trate a continuación de aclarar la definición apelando al análisis leibniziano, ilustra bien que ha captado la función pedagógica que Wolff le asigna al análisis conceptual.

\section{LA DISTINCIÓN ANÁLISIS-SÍNTESIS EN LAS LECCIONES DE MUTIS SOBRE LA GEOMETRÍA DE DESCARTES}

En sus lecciones de la cátedra consagradas a enseñar la geometría analítica Mutis hace nuevos planteamientos sobre el método de análisis y síntesis con respecto a los mencionados anteriormente. Comienza diciendo que expondrá el tema de manera «fácil y perceptible» en atención a los alumnos que emprenden por primera vez su estudio. En la más fiel aplicación de la «regla del análisis» al acto de enseñar, dice que procederá «examinando por partes, y poniendo en cada lugar, todo lo que me parece útil para hacer inteligible su doctrina». ${ }^{28}$

Esto corresponde a un propósito pedagógico de no fundamentar la enseñanza directamente en la presentación del original, ya que siendo la Geometría la más importante de las obras de Descartes, es la de más difícil lectura. Por otra parte, en el discurso del Método matemático, Mutis se había referido a la «regla de síntesis» interpretándola igualmente en un contexto pedagógico: «[Que] todas las proposiciones cuyas verdades no constan a primera vista por la significación y percepción de los mismos términos con que se enuncian, se hayan de probar demostrando muchas verdades, y por medio de las definiciones supuestas, los axiomas

\footnotetext{
28 Arboleda, «Matemáticas, Cultura y Sociedad en Colombia», 52 y 66. En este trabajo nos referimos de manera incidental al manuscrito con las notas de la enseñanza de Mutis de la geometría cartesiana, titulado Comentarios a la Geometría de Descartes. Este manuscrito se conserva en el Fondo Mutis del Real Jardín Botánico de Madrid. Recientemente Molina realizó su completa identificación, así como el primer estudio histórico pormenorizado del mismo, en el marco de su tesis doctoral: Molina, «José Celestino Mutis and the appropriation of Newton's experimental physics in New Granada», 118-124. Agradecemos a doña Irene Fernández de Tejada de Garay, Unidad de Archivo, Real Jardín Botánico, CSIC, el habernos facilitado una copia de este manuscrito [RJB III 7, 1, 5, ff. 397r-416v] para la preparación del presente trabajo.
} 
concedidos y las proposiciones ya demostradas». ${ }^{29}$ Mutis advierte que no es su intención hacer ningún elogio a Descartes, primero porque no lo podría hacer al nivel de lo que ese gran hombre se merece, pero ante todo porque «el mejor elogio será el explicar bien su Geometría». Parece que Mutis quiso así dejar en claro a sus alumnos que comprender al geómetra es una cosa y combatir al filósofo de sistema es otra.

Para la realización del primer propósito — explicar bien la geometría cartesiana y facilitar su comprensión entre sus alumnos- Mutis probablemente aprovechó las Lecciones de matemáticas de La Caille, ${ }^{30}$ un texto de reconocida utilidad pedagógica en su época, y al cual se refiere en varios de sus escritos. La Caille le habría aportado una visión menos especulativa y más orientada al abordaje del método analítico en la enseñanza del álgebra y la geometría analítica. Anotemos que existen evidencias de que este texto fue consultado en otros momentos de la cátedra y en la formación autodidacta por varios miembros de la élite criolla a lo largo de los decenios siguientes; incluso ya bien entrada la república —alrededor de los años 1850— Lino de Pombo lo utilizó en la enseñanza de la geometría analítica en el Colegio Militar. ${ }^{31}$

Retornemos al manuscrito de Mutis titulado Comentarios a la Geometría de Descartes [RJB III 7, 1, 5, ff. 397r-416v]. Gracias al trabajo de Molina $^{32}$ hoy sabemos que estas «notas de enseñanza» de la geometría cartesiana por parte de Mutis en la cátedra del Colegio del Rosario, corresponden efectivamente a la traducción inédita al español, hecha por él mismo, de los Comentarios que Rabuel consagró a la Geometría de Descartes. ${ }^{33}$ Hay que advertir que la traducción de Mutis no incluye,

\footnotetext{
${ }_{29}$ Mutis, Pensamiento científico y filosófico, 126. Ver igualmente Arboleda, «Matemáticas, Cultura y Sociedad en Colombia», 53.

${ }^{30}$ Nicolas-Louis de La Caille, Leçons Élémentaires de Mathématiques ou Éléments d'Algebre et de Géométrie (Paris: Guerin, 1747).

31 Lino de Pombo, Lecciones de jeometría analítica (Bogotá: Imprenta El día, 1850) y Luis Carlos Arboleda, "La introducción del método analítico en la enseñanza de las matemáticas en Colombia», Revista Paradigma 39 (2018): 202-222.

${ }^{32}$ Molina, «José Celestino Mutis and the appropriation of Newton's experimental physics in New Granada», 118-124.

33 Claude Rabuel, Commentaires sur la géométrie de M. Descartes (Lyon: M. Duplain, 1730). Un ejemplar de esta obra — como otras de la biblioteca de Mutis referidas en este trabajo- se conserva actualmente en la Biblioteca Nacional de Colombia.
} 
entre otros apartes, el texto sobre el procedimiento empleado en la Geometría en la solución del problema de Pappus. Como hemos aclarado anteriormente, fue precisamente aquí donde Descartes utilizó el análisis para obtener el principio de la solución; es decir, la curva algebraica cuya fórmula general es la ecuación de segundo grado; para luego deducir de esta ecuación, mediante un procedimiento de síntesis, las secciones cónicas que satisfacen la solución y finalmente construirlas con regla y compás.

Por otra parte, Mutis solo traduce 8 de las 12 Reglas generales para la solución de los Problemas en las cuales Rabuel se propone sistematizar la explicación de Descartes sobre «Cómo se ha de llegar a las igualaciones que sirven para resolver los Problemas». No traduce en particular la Regla XII en donde Rabuel comenta precisamente la noción de fórmula general; es decir, aquella que, manteniendo una estrecha relación con el método analítico,

hace evidente todas las combinaciones que pueden tener los términos y los signos de una clase de ecuaciones, y que involucran todos los casos que se pueden presentar en la resolución o construcción de un Problema. Aquella que expresa los distintos grados de las curvas a las cuales se da el mismo nombre. ${ }^{34}$

No obstante lo anterior, la traducción contiene las reglas del I al VIII en donde Rabuel tematiza las explicaciones de Descartes sobre su método de resolución geométrica mediante el análisis algebraico. ${ }^{35}$

\section{LAS VARIACIONES DEL ENFOQUE NEWTONIANO SOBRE EL MÉTODO ANALÍTICO EN LA CÁTEDRA DE MUTIS}

En el aparte anterior hemos señalado que para Mutis fue claro, desde los primeros años de la cátedra, que enseñar la geometría de Descartes no era incompatible con su empeño en rebatir su filosofía de sistema. Precisamente en las lecciones de 1764 sobre los Elementos de la filosofía

\footnotetext{
34 Traducción de L. C. A.

35 Ver Sébastien Maronne, «Les Commentaires sur la Géométrie de M. Descartes (1730) de Claude Rabuel», en Autour de Descartes et Newton. Le paysage scientifique lyonnais dans le premier XVIIIe siècle, eds. Pierre Crépel y Christophe Schmit (Paris: Hermann, 2017), 111-161 y 349-355 (Annexe).
} 
natural, ${ }^{36}$ Mutis consagra varios apartes a rebatir el método de los escolásticos de construir sistemas globalmente explicativos «inventados por la fuerza del ingenio». Argumentando contra las pretensiones cartesianas de deducir la explicación de los efectos a partir de causas formuladas dentro de sistemas a priori, Mutis vuelve a referirse al método analítico-sintético, pero ahora no en la perspectiva logicista, sino relacionándolos con su interpretación newtoniana en el estudio de la naturaleza. Todo indica que se basó en la «cuestión 31 » de la Óptica de Newton, ${ }^{37}$ aunque está por esclarecerse cuál de las ediciones utilizó entre las que se encuentran en los fondos Mutis y de libros raros y curiosos de la Biblioteca Nacional de Colombia en Bogotá.

En la continuación de este aparte de los Elementos que más nos interesa, Mutis afirma que el estudio de la naturaleza de acuerdo con el método de análisis y síntesis es el más seguro y que por ello permite abandonar para siempre todo tipo de disputas. Se comienza, dice Mutis, por el examen de las causas o efectos de los fenómenos para luego pasar al descubrimiento de sus potencias o causas. Observemos que Mutis no utiliza la equivalencia que establece Newton al comienzo de la «cuestión 31 » entre las terminologías de "análisis y síntesis» y su equivalente «resolución y composición». Mutis omite igualmente la consideración de Newton sobre el papel de la inducción en la obtención de conclusiones generales a partir de los experimentos. Su explicación de la idea de Newton sobre análisis y síntesis se reduce a lo siguiente:

[Newton] estableció [...] que de las causas particulares se fuera subiendo a otras más generales; y de éstas finalmente a las más generales entre todas. Este es el método analítico. Después de haber descubierto estas causas se debe bajar por un orden contrario, considerándolas ya como principios establecidos para explicar por este medio las causas menos generales, y después los fenómenos que son sus consecuencias; haciendo ver de este modo

\footnotetext{
36 José Celestino Mutis, «Elementos de la filosofía natural, que contienen los principios de la física demostrados por las matemáticas y confirmados con observaciones y experiencias: dispuestos para instruir a la juventud en la doctrina de la filosofía newtoniana en el Real Colegio del Rosario de Santa Fe de Bogotá en el Nuevo Reino de Granada, año de 1764», en Mutis, Pensamiento científico y filosófico, 43-68. Citado en este trabajo como Elementos.

37 Isaac Newton, Opticks, or, A Treatise of the Reflections, Refractions, Inflections, and Colours of Light (New York: Prometheus Books, 2003).
} 
la solidez y firmeza de estas explicaciones. Este es el método sintético. $^{38}$

A continuación, Mutis advierte que en la aplicación de este método en cualquier materia de las matemáticas o de la física - los términos originalmente empleados por Newton son «física natural» $\mathrm{y}$ «física experimental»-, hay que tener en cuenta que existe una jerarquía epistemológica del momento de análisis con respecto a la síntesis. Esta argumentación viene a reforzar el propósito de los Elementos de presentar la filosofía natural en un contexto de crítica radical a la vieja filosofía, pues la primacía del análisis ofrece la garantía de reconocer y utilizar principios que efectivamente existen en la naturaleza y, por consiguiente, permite abandonar sistemas que, aun habiendo sido compuestos a través de síntesis laboriosas, resultan ilusorios.

En la anterior interpretación sobre el uso newtoniano del método de análisis y síntesis en la filosofía natural, se sostiene que Mutis se inspiró, como hemos dicho, en la aproximación experimentalista a este método que aparece en la «cuestión 31» de la Óptica. Conviene hacer una aclaración a este respecto. Los biógrafos de Mutis coinciden en señalar que los Elementos o lecciones de Mutis de 1764 sobre la filosofía natural corresponden al enfoque experimental adquirido en la primera parte de su formación en medicina en Cádiz, durante los años 1753-1757, bajo la orientación de Pedro Virgili (1699-1776). Como se sabe, Virgili fue el promotor de la apropiación en España de los principios de la física newtoniana aplicados a la explicación de los fenómenos fisiológicos. ${ }^{39}$ Esta cuestión y otras relacionadas con la formación de Mutis y su actividad en la enseñanza concretamente de la física experimental en la Nueva Granada, han sido examinadas por Molina en su tesis desde una perspectiva más documentada y completa que seguramente será objeto de nuevas reflexiones en la materia. ${ }^{40}$

\footnotetext{
${ }_{38}$ Mutis, Pensamiento científico y filosófico, 51.

39 Emilio Quevedo, «Mutis y la medicina», en Mutis y la Real Expedición Botánica del Nuevo Reino de Granada, ed. María del Pilar San Pío Alarden (Barcelona: Villegas/Lunwerg Editores, 1992), 77 y 97.

40 Molina, «José Celestino Mutis and the appropriation of Newton's experimental physics in New Granada», 76-83; se refiere al interesante aparte titulado «Pedro Virgili, la iatromecánica y su influencia en Mutis».
} 
También se ha planteado que por la época de su formación en Cádiz, Mutis pudo haber frecuentado la Asamblea amistosa literaria dirigida por Jorge Juan en Cádiz, o al menos estuvo al tanto de los trabajos científicos modernos que en ella se discutían, así como también habría participado en las actividades del recién creado Observatorio astronómico de San Fernando. ${ }^{41}$ Posteriormente, entre 1757 y 1760, Mutis estuvo en condiciones de aprovechar su estancia como médico en la corte de Madrid para fundamentar su formación científica particularmente en matemáticas y física, a través del estudio de las obras de los físicos experimentalistas entonces más reconocidos: los holandeses Willen's Gravesande y Pieter van Musschenboek, y los franceses Sigaud de la Fond y Jean Antoine Nollet, obras estas que enseñó y difundió en la primera etapa de la cátedra en la Nueva Granada. ${ }^{42}$

De manera que el texto Elementos corresponde a la primera parte de la formación newtoniana de Mutis con un acercamiento a la nueva física desde la experiencia sensible y de acuerdo con el enfoque de la Óptica, la cual se constituyó desde su publicación en 1704 en un poderoso medio de penetración y aceptación de Newton en el continente. Cuando esta etapa experimental estuvo suficientemente consolidada, tanto por los esfuerzos de los primeros newtonianos (los experimentalistas holandeses y franceses antes citados) como de cartesianos como Malebranche, Mairan y Privat de Molières, entonces se hizo posible la conformación de un consenso sobre la obra paradigmática de los Principia.

Este ciclo histórico se verificó de cierta manera en la Nueva Granada. A la primera etapa de enseñanza de la física experimental en los inicios de la cátedra de matemáticas en el Colegio del Rosario, seguiría un periodo de cualificación de los estudios de la física matemática newtoniana, llegando a su madurez en los años 1770 con la traducción al castellano de los Principia de Newton en la célebre edición latina de

\footnotetext{
${ }^{41}$ Francisco González de Posada, «José Celestino Mutis médico, y la ciencia fundamental de su tiempo en España», Real Academia de Medicina de Cantabria 26 (2008), 45-46.

42 Arboleda, «Matemáticas, Cultura y Sociedad en Colombia», 71-91. Ver los nuevos elementos históricos que aporta Molina en el capítulo 4 de su tesis en cuanto a la formación y enseñanza de Mutis de la física newtoniana, particularmente en el enfoque de los experimentalistas holandeses: Molina, «José Celestino Mutis and the appropriation of Newton's experimental physics in New Granada», 134-212.
} 
Leseur \& Jacquier. ${ }^{43}$ La traducción de la obra canónica de la nueva física fue una tarea de enormes proporciones, aunque lamentablemente inacabada e inédita, que Mutis realizó en colaboración de algunos de sus alumnos más aventajados en la cátedra del Rosario, en un momento de álgidas discusiones contra los detractores del copernicanismo y las teorías heliocéntricas. ${ }^{44}$

No olvidemos que Leseur y Jacquier enriquecieron la edición de los Principia con numerosos comentarios históricos y científicos, particularmente útiles para quienes, estando alejados de los centros académicos europeos, como era el caso de Mutis y sus alumnos, requerían informarse sobre el desarrollo y el estado del arte de las matemáticas y la física hasta la primera parte del siglo XVIII. Así mismo, estaban dirigidos a facilitarle al lector la comprensión del verdadero estilo newtoniano de matematización de la naturaleza, y su diferencia con respecto al enfoque experimentalista ilustrado. Por ejemplo, al traducir los corolarios I y II a las leyes del movimiento de Newton en la edición latina, Mutis también tradujo los comentarios de los editores sobre la modelación de la composición y resolución de las fuerzas y movimientos por medio de la ley del paralelogramo.

Después de examinar las técnicas y argumentos de las demostraciones matemáticas de los corolarios, Leseur y Jacquier destacan las ingeniosas y precisas experiencias con las cuales Gravesande había confirmado la exactitud de tales demostraciones. Este bien pudo ser uno de aquellos momentos de la traducción en los cuales la apropiación intelectual de la obra canónica, que se pone en juego en este ejercicio, condujo al reconocimiento por parte de Mutis y de sus alumnos de que la cultura anterior formada en los textos de los divulgadores experimentales de Newton tenía su razón de ser en la teoría expuesta en los Principia.

\footnotetext{
43 Thomas Leseur y François Jacquier, Philosophiae naturalis principia mathematica auctore Isaaco Newtono eq aurato: perpetuis commentaris illustrate (Genevae: Barrillot, 1739-1742).

${ }^{44}$ Luis Carlos Arboleda, «Newton en Nueva Granada. Anticartesianismo y matematización de la realidad en la traducción mutisiana de los Principia», en Mutis y la Real Expedición Botánica del Nuevo Reino de Granada, ed. María del Pilar San Pío Alarden, (Barcelona: Villegas/Lunwerg Editores, 1992), 33-47. Ver en Arboleda, «Matemáticas, Cultura y Sociedad en Colombia», el capítulo 4 (93-114) sobre la traducción de los Principia, y el capítulo 5 (115-143) sobre las polémicas del copernicanismo.
} 
Por cierto, el papel de Mutis en la institucionalización de la física newtoniana en Nueva Granada fue reconocido de manera temprana por los viajeros científicos europeos que exploraron nuestro territorio, en particular por Humboldt quien lo divulgó en Europa a través de su Diario de viaje $\mathrm{y}$, de manera especial, en el obituario consagrado al gaditano en la célebre Biographie Universelle de Michaud, en donde escribió lo siguiente:

Como profesor de matemáticas del Colegio Mayor de Nuestra Señora del Rosario, difundió las primeras nociones del verdadero sistema planetario en Santa Fe. Los dominicos no vieron sin inquietud que «las herejías de Copérnico» profesadas ya por Bouguer, Godin y La Condamine, en Quito, penetraran a la Nueva Granada; pero el virrey protege a Mutis de los monjes que querían que la tierra permaneciera inmóvil. Estos se acostumbraron poco a poco a lo que llamaban todavía «las hipótesis de la nueva filosofía».45

Retornando a nuestro tema principal, recordemos que tanto en los Principia como en los comentarios de Leseur y Jacquier aparecen consideraciones sobre la concepción newtoniana del método de análisis-síntesis que seguramente no escaparon a la atención de sus lectores en Santafé de Bogotá, tanto más por la insistencia de Mutis de comprender a fondo el «verdadero método de filosofar». Así, por ejemplo, desde el Prefacio, Newton advierte que la preocupación central de su filosofía consiste en investigar las fuerzas de la naturaleza a partir de los fenómenos del movimiento, lo cual corresponde al momento del análisis. Luego, a partir del conocimiento de los principios de estas fuerzas, se pasa a demostrar el resto de los fenómenos; es decir, del momento del análisis sigue el momento de la síntesis. ${ }^{46}$

Así mismo, en el corolario II a las leyes del movimiento antes mencionado se encuentran varios comentarios de Leseur y Jacquier sobre la

\footnotetext{
45 Alexander von Humboldt, "Mutis Don Joseph Celestino», en Biographie Universelle Ancienne et Moderne, ed. Michaud (Paris: Desplaces, 1823), vol. 29, 658.

46 Ver el aparte «Mutis y la matematización de la realidad física» en Arboleda, «Newton en Nueva Granada. Anticartesianismo y matematización de la realidad en la traducción mutisiana de los Principia», 39-42. Ver Sebastián Molina, "Aspectos metodológicos de la demostración de la fuerza en los Principia de Newton», Praxis Filosófica 39 (2014): 67-92
} 
composición de fuerzas en donde se revela la complejidad del método de la filosofía natural. La traducción del aparte sobre la experiencia del sistema de fuerzas asociado al movimiento de una rueda debe haber aproximado mucho más a Mutis y a sus alumnos a la comprensión del concepto de matematización newtoniana de la naturaleza, en comparación con la primera parte de la enseñanza de la cátedra consagrada a divulgar el enfoque experimentalista de la nueva física.

Una lectura a fondo del comentario de los editores sobre el estudio de este sistema de fuerzas, como la que tuvo que haber hecho Mutis con la traducción, pone en cuestión cualquier imagen simplificadora de la naturaleza y uso del método de análisis-síntesis. En particular, le permitió dejar en claro que lo decisivo en el método de Newton no era la generalización por inducción de los resultados más simples de la experiencia, sino, como dice Cohen, «un intercambio entre la simplificación e idealización de las situaciones que se dan en la naturaleza y sus análogos en el dominio matemático. De este modo, Newton pudo producir un sistema matemático y unos principios matemáticos que luego se aplicarían a la filosofía natural».47.

\section{MUTIS LECTOR DE VERSIÓN MODERNA DEL MÉTODO ANALÍTICO EN LOS ELEMENTOS DE BAILS}

Otro evento determinante en la adopción del método analítico en la cátedra de matemáticas del Colegio del Rosario fue la introducción en la enseñanza de las obras de Benito Bails, tanto los Elementos de Matemática ${ }^{48}$ sin duda el compendio enciclopédico de las matemáticas más importante de España en el siglo XIX, como en su versión abreviada de los Principios de Matemática. ${ }^{49}$

El tomo segundo de los Elementos de Bails es un tratado de álgebra. Se estudian esencialmente los métodos de resolución de ecuaciones hasta de cuarto grado y su aplicación en el tratamiento algebraico de

\footnotetext{
47 Ver la ya célebre caracterización del «estilo matemático» de Newton en I. Bernard Cohen, La revolución newtoniana y la transformación de las ideas científicas (Madrid: Alianza, 1983), 71174.

${ }^{48}$ Benito Bails, Elementos de Matemática (Madrid: Joachim Ibarra, 1772-1783).

${ }^{49}$ Benito Bails, Principios de Matemática (Madrid: Vda. de Ibarra, 1776).
} 
problemas de la geometría; en ello consiste el método analítico para Bails. Análisis es el «arte que enseña los métodos para resolver por el cálculo algebraico las cuestiones que se pueden proponer acerca de las cantidades», y Analistas aquellos matemáticos que se dedican a este ramo o aplicación del cálculo algebraico.

En el tomo tercero se exponen los fundamentos del «análisis cartesiano»; es decir, se caracterizan las curvas algebraicas, particularmente las cónicas, por el método de coordenadas, y se determinan sus propiedades mediante el cálculo diferencial e integral. Concluye el tomo con una presentación de ecuaciones diferenciales y un pequeño tratado de trigonometría esférica.

La introducción de Bails en la enseñanza de las matemáticas en Colombia es un hecho de significativa importancia, ya que, comparada con la influencia de la obra de Wolff en su etapa fundacional de la cátedra del Rosario, conllevó una transformación en el enfoque pedagógico y en los contenidos a enseñar. Mutis recomienda a Bails en el Plan provisional para la enseñanza de las matemáticas en el Colegio de Nuestra Señora del Rosario de $1785.5^{50}$

En la presentación del plan al virrey Caballero y Góngora, Mutis explica que si al comienzo de la cátedra en 1762 había preferido el compendio y el curso completo de Wolff, fue por «la dilatada experiencia de enseñar de aquel profesor» y porque estas eran «obras excelentes en su tiempo y modelo de las que posteriormente se han publicado». Pero los avances posteriores de las teorías matemáticas, agrega, han evidenciado que las obras de Wolff son «en cierto modo defectuosas» y que ya no convienen a la instrucción. ${ }^{51}$

La opinión puede parecer desmesurada en cuanto a ubicar el compendio de Bails como una de las mejores obras de Europa, a no ser que se refiera al hecho de que, como lo reconoce el propio Bails en su prólogo, en su realización extractó y copió de las obras clásicas y modernas, en particular de los cursos recientes de Bézout y Cramer, aquello que de acuerdo

\footnotetext{
50 Mutis, Pensamiento científico y filosófico, 117-124.

${ }^{51}$ Mutis, Pensamiento científico y filosófico, 119-120.
} 
con los propósitos pedagógicos del plan le pareció más indicado, «enlazando con todo esmero los pedazos para la formación de un tratado».52

Pero Mutis no se equivoca en su percepción de la inmediata difusión que la primera edición y posteriores reimpresiones de Bails tuvieron en España y en América, y su influencia en la preparación de un nuevo espíritu científico en las ciencias matemáticas. Esta acogida venía a responder a los nuevos derroteros de la institucionalización de la enseñanza en España y sus colonias, y a las expectativas de formación matemática centrada ya no tanto en una curiosidad ilustrada por las matemáticas, como en la búsqueda de una comprensión integral y formalizada de las teorías más avanzadas del momento. Todo ello enfocado en aquellos saberes que, como decía Bails en el prólogo general de la obra, satisfacen las «empresas de universal utilidad», y coadyuvan al interés natural de «sacrificar la especulación a la práctica».53

Por cierto, en el prólogo Bails pone las Lecciones de La Caille como ejemplo de lo que él entendía como moderna concepción de un «curso completo de matemáticas», pero lamenta que ellas fueran extremamente concisas y que hubieran omitido importantes temas, sobre todo en matemáticas puras. Sin embargo, reconoce que esta concisión no obedecía a impreparación de su autor, sino a un empeño consciente de solo exponer en el texto los fundamentos de las materias a enseñar, dejando al profesor la tarea de complementarlas y explicarlas en la enseñanza en el aula. ${ }^{54}$

Según Bails, esta concepción pedagógica buscaba reemplazar el dictado en la enseñanza de las matemáticas. Se trataba de impedir que, por mantener fija la atención en el dictado de las materias más que en su comprensión, los alumnos cometieran errores en la copia apresurada, concretamente de cálculos y fórmulas complicadas. Estos errores eran incluso frecuentes en la copia más esmerada de las figuras y diagramas con los cuales se ilustraban los contenidos. ${ }^{55}$

\footnotetext{
${ }_{52}$ Bails, Elementos de Matemática, 1: xiii, xix.

${ }_{53}$ Bails, Elementos de Matemática, 1: xiv.

${ }^{54}$ Bails, Elementos de Matemática, 1: v-vi.

${ }_{55}$ Bails, Elementos de Matemática, 1: vii.
} 
En el tomo segundo sobre el álgebra encontramos otra reflexión pedagógica de Bails sobre el método analítico entendido, en el contexto intelectual del momento, como aplicación del álgebra a la geometría. Bails informa en el prólogo que en su presentación utilizó el Comentario de Rabuel a la Geometría de Descartes, ${ }^{56}$ precisamente la obra que Mutis estudió y tradujo en cierto momento de su enseñanza en la cátedra del Rosario. También se refiere a las dificultades que enfrentan quienes se inician en la aplicación del método analítico (algebraico) en la resolución de problemas geométricos; concretamente en cuanto a introducir la doble escritura cartesiana para designar los segmentos dados y distinguirlos de los segmentos desconocidos a partir de la configuración geométrica del problema, y poder así avanzar en la construcción de la ecuación final.

Recordemos que Descartes reconoce esta dificultad en la Geometría, al señalar que su solución debe abordarse en el orden más natural para mostrar la manera de relacionar unos segmentos con otros. En su enseñanza de la geometría cartesiana, Mutis se encontraba bien al tanto de esta dificultad y disponía de los elementos propuestos por Rabuel para superarla en la cátedra en términos de sus «12 reglas». ${ }^{57}$ Años más tarde, al publicarse el tomo segundo de los Elementos, Mutis se encontrará de nuevo con las técnicas y procedimientos que Bails ofrece al calculador o analista para vencer tal dificultad, los cuales no lo eximen de un esfuerzo laborioso e inteligente de construcción analítica, como advierte Rabuel en el Comentario:

Más es fortuna que destreza escoger con acierto las líneas y empezar como conviene el cálculo. Cuestión hay que se resuelve con suma facilidad siguiendo un rumbo, la cual sería trabajosa, y acaso imposible de resolver, siguiendo otro distinto. Por lo que, siempre que un camino parezca largo, o muy penoso, será prudente buscar otro, u otros muchos, si fuera necesario. No os

\footnotetext{
${ }^{56}$ Bails, Elementos de Matemática, 2: vi.

57 Descartes, Geometry, 8. Se trata de la bien conocida cita del libro 1 sobre el uso de ecuaciones para resolver problemas, la cual aparece como Sección IV de la traducción mutisiana del Comentario de Rabuel, bajo el título «Como se ha de llegar a las igualaciones que sirven para resolver los Problemas». A este aparte original de la Geometría de Descartes siguen en la traducción las «12 Reglas generales para la resolución de los Problemas» de Rabuel, las cuales, como se explicó antes, se reducen a ocho en el manuscrito [RJB III 7, 1, 5, ff. 397r-416v].
} 
dejéis alucinar de las resoluciones breves y despejadas que leyereis en las obras impresas, donde se encuentran cuestiones resueltas con tal brevedad y elegancia, que luego se entiende su resolución, a la cual no llegó su autor, sino después de muchísimo trabajo, y de haberla buscado en vano por muchos caminos. ${ }^{58}$

\section{Nota sobre el autor}

Luis Carlos Arboleda Aparicio es Profesor Emérito de la Universidad del Valle, Cali, Colombia, e Investigador Emérito de Colciencias. Actualmente se desempeña como profesor-investigador en el área de Educación Matemática del Instituto de Educación y Pedagogías. Es miembro del Grupo de Historia y Filosofía de la Práctica Matemática, y del Grupo Nación, Cultura y Memoria de esa institución, y coordinador del Grupo de Historia y Filosofía de la Ciencia de la Academia Colombiana de Ciencias Exactas, Físicas y Naturales de Colombia. Realizó sus estudios de Licenciatura en Matemáticas y Física, primero en la Universidad del Valle y luego en la Universidad Santiago de Cali. Hizo una Especialización en Lógica y Epistemología de la Ciencia en el Instituto de Historia de las Ciencias de la Academia de Ciencias de Varsovia. Adelantó su formación de Maestría y Doctorado en el Programa de Historia de las Culturas, los Saberes y la Educación en la Escuela de Altos Estudios en Ciencias Sociales de París. Su tesis doctoral versó sobre los Orígenes de la Topología General y el Análisis Funcional. Desarrolló una investigación posdoctoral en Historia Social de las Ciencias en el Centro de Estudios Históricos del CSIC-Madrid, sobre "La Traducción Inédita de los "Principia" de Newton por Mutis en la Nueva Granada circa 1772". Sus líneas de investigación son: Historia y Filosofía de las Matemáticas, Historia en la Educación Matemática e Historia de las Matemáticas en Colombia, y Apropiación y uso de la Historia de las Matemáticas en la Formación de profesores.

\footnotetext{
58 Esta es la traducción que hace Bails, Elementos de Matemática, 2, del texto original que aparece en Claude Rabuel, Commentaires sur la géométrie de M. Descartes (Lyon: M. Duplain, 1730), 22 (nota de pie de página).
} 


\section{REFERENCIAS}

Arboleda, Luis Carlos. «Newton en Nueva Granada. Anticartesianismo y matematización de la realidad en la traducción mutisiana de los Principia». En Mutis y la Real Expedición Botánica del Nuevo Reino de Granada, editado por María del Pilar San Pío Alarden, 33-47. Barcelona: Villegas/Lunwerg Editores, 1992.

Arboleda, Luis Carlos. "Matemáticas, Cultura y Sociedad en Colombia». En Historia Social de la Ciencia en Colombia, editado por Emilio Quevedo, tomo II, 13-172. Bogotá: Colciencias, 1993.

Arboleda, Luis Carlos. «El análisis cartesiano en la solución del problema de Pappus y la introducción de las curvas algebraicas». En Asociación Colombiana de Matemática Educativa $13^{\circ}$ Encuentro, editado por Gilberto Obando Zapata, 764-776. Medellín: Editorial Universidad de Medellín, 2013.

Arboleda, Luis Carlos. «La introducción del método analítico en la enseñanza de las matemáticas en Colombia». Revista Paradigma 39 (2018): 202-222.

Bails, Benito. Elementos de Matemática. 10 vols. Madrid: Joachim Ibarra, 17721783.

Bails, Bails. Principios de Matemática. 3 vols. Madrid: Vda. de Ibarra, 1776.

Cantù, Paola. «Mathematics. Systematical Concepts». En Handbuch Christian Wolff, editado por Robert Theis y Alexander Aichele, 357-380. Wiesbaden: Springer, 2018.

Cohen, I. Bernard. La revolución newtoniana y la transformación de las ideas científicas. Madrid: Alianza, 1983.

Crépel, Pierre y Christophe Schmit (eds.). Autour de Descartes et Newton. Le paysage scientifique lyonnais dans le premier XVIIIe siècle. Paris: Hermann, 2017.

Descartes, René. The Geometry of René Descartes with a facsimile of the first edition. Translated from the French and Latin by David Eugene Smith and Marcia L. Lattan. New York: Dover, 1954.

Gardies, Jean-Louis. Qu'est-ce que et pourquoi l'analyse? Essai de définition. Paris: Vrin, 2001.

González de Posada, Francisco. José Celestino Mutis, médico, y la ciencia fundamental de su tiempo en España. Santander: Real Academia de Medicina de Cantabria, 2008.

Hilbert, David. The Foundations of Geometry. Authorized translation by E. J. Townsend. Reprint edition. La Salle: The Open Court Publishing Co., 1950.

Hintikka, Jaakko y Unto Remes. The Method of Analysis. Its Geometrical Origin and Its General Significance. Dordrecht: Reidel, 1974.

Humboldt, Alexander von. «Mutis Don Joseph Celestino». En Biographie Universelle Ancienne et Moderne, editado por Michaud, nouvelle édition, vol. 29: 658-662. Paris: Desplaces, 1823. 
Klein, Jacob. Greek Mathematical Thought and the Origin of Algebra. New York: Dover, 1968.

La Caille, Nicolas-Louis de. Leçons Élémentaires de Mathématiques ou Élémens d'Algebre et de Géométrie. Paris: Guerin, 1747.

Leseur, Thomas y François Jacquier. Philosophiae naturalis principia mathematica auctore Isaaco Newtono eq aurato: perpetuis commentaris illustrate. Genevae: Barrillot, 1739-1742.

Maronne, Sébastien. «Les Commentaires sur la Géométrie de M. Descartes (1730) de Claude Rabuel». En Autour de Descartes et Newton. Le paysage scientifique lyonnais dans le premier XVIIIe siècle, editado por Pierre Crépel y Christophe Schmit, 111-161 y 349-355 (Annexe). Paris: Hermann, 2017.

Molina, Sebastián. «Aspectos metodológicos de la demostración de la fuerza en los Principia de Newton». Praxis Filosófica 39 (2014): 67-92.

Molina, Sebastián. "No hay reino que no sea newtoniano”: José Celestino Mutis and the appropriation of Newton's experimental physics in New Granada (1762-1804)». PhD diss., Università degli Studi di Torino, 2018.

Mutis, José Celestino. Pensamiento científico y filosófico de José Celestino Mutis. Editado por Gonzalo Hernández de Alba. Bogotá: Fondo Cultural Cafetero, 1982.

Newton, Isaac. Opticks, or, A Treatise of the Reflections, Refractions, Inflections, and Colours of Light. New York: Prometheus Books, 2003.

Otte, Michael y Marco Panza (eds.). Analysis and Synthesis in Mathematics. Dordrecht: Kluwer, 1997.

Pombo, Lino de. Lecciones de jeometría analítica. Bogotá: Imprenta El día, 1850.

Quevedo, Emilio. «Mutis y la medicina». En Mutis y la Real Expedición Botánica del Nuevo Reino de Granada, editado por María del Pilar San Pío Alarden, 77-97. Barcelona: Villegas/Lunwerg Editores, 1992.

Rabuel, Claude. Commentaires sur la géométrie de M. Descartes. Lyon: M. Duplain, 1730.

Wolff, Christian. Elementa matheseos universae. Qui commentationem de methodo mathematica, arithmeticam, geometriam, trigonometriam planam, et analysin tam finitorum, quam infinitorum complectitur. 2 vols. Halle: Magdeburgicae, 1713-1715.

Wolff, Christian. Cours de mathématique contenant toutes les parties de cette science mises à la portée des commençants, traduit en françois e augmenté considérablement, 3 tomes. Paris: Jombert, 1757.

Wolfii, Christiani [Christian Wolff]. Elementa matheseos universae. Editio novissima, multo auctior et Correctior, 5 tomos. Genevae: Henricum-Albertum Gosse, 1743-1752. 